\title{
Estimating Mobile Traffic Demand using Twitter
}

\author{
Bowei Yang ${ }^{1}$, Weisi Guo ${ }^{2 *}$, Bozhong Chen ${ }^{3}$, Guangpu Yang ${ }^{3}$, Jie Zhang ${ }^{3,4}$
}

\begin{abstract}
In this paper, the authors show that structured social media data can act as an accurate predictor for wireless data demand patterns at a high spatial-temporal resolution. A casestudy is performed on Greater London covering a $5000 \mathrm{~km}^{2}$ area. The data used includes over 0.6 million geo-tagged Twitter data, over 1 million mobile phone data demand records, and UK census data. The analysis shows that social media activity (Tweets/s $n$ ) can accurately predict the long-term traffic demand for both the uplink and downlink channels. The relationship between social media activity and traffic demand obeys a power law and the model explains for over $71-79 \%$ of the variance in real traffic demand. This is a significant improvement over existing methods of long-term traffic prediction such as census population data $\left(R^{2}=0.57\right)$. The authors also show that social media data can also forward predict short-term traffic demand for up to 2 hours on the same day and for the same time in the following 2-3 days.
\end{abstract}

\section{INTRODUCTION}

Over the past few years, large volumes of data has been transforming businesses to deliver higher precision and more personalised services. Fine-grain traffic data can transform the business model of cellular network operators by enabling the deployment of small-cells [1]. Historically, Call Detail Record (CDR) based research, has yielded useful macroscopic statistical models on the spatial-temporal pattern of traffic demand. For example: the number of active users in a BS is $\sim \operatorname{Pois}($. distributed and the $3 \mathrm{G}$ traffic demand per user session is $\sim \log -\mathrm{N}($.$) distributed [2]-[4]$. As operators seek to deploy small-cells to efficiently scale the overall network capacity and target traffic hotspots or signal blind-spots, there is a need to move from macroscopic traffic models to microscopic traffic modeling.

Small-cell deployment needs to consider a number of important parameters [5], [6]: 1) high resolution traffic demand data over a small coverage radius $(10-25 \mathrm{~m}), 2)$ interference with adjacent cells, and 3) high resolution signal propagation modeling that is sensitive to building architecture and materials. In this paper, we focus on addressing challenge 1), which has received relatively little attention, but is crucial to the economic feasibility of the small-cell business. Traditionally, traffic is measured using CDR data, but as CDR is typically aggregated over the relatively large coverage area of macro Base Stations (BSs). Even with cooperative localization and antenna information, the accuracy is not sufficient for small

\footnotetext{
${ }^{1}$ School of Aeronautics and Astronautics, Zhejiang University, China. ${ }^{2}$ School of Engineering, University of Warwick, UK. ${ }^{3}$ Department of Electronic and Electrical Engineering, University of Sheffield, UK. ${ }^{4}$ RANPLAN, Cambridge, UK. This research is partially supported by EU H2020 "DECACE" project and the National Natural Science Foundation of China (NSFC) grant No. 61501399 and 61272467, and the EPSRC Centre for Doctoral Training in Urban Science and Progress (EP/L016400/1). * corresponding author email: weisi.guo@warwick.ac.uk
}

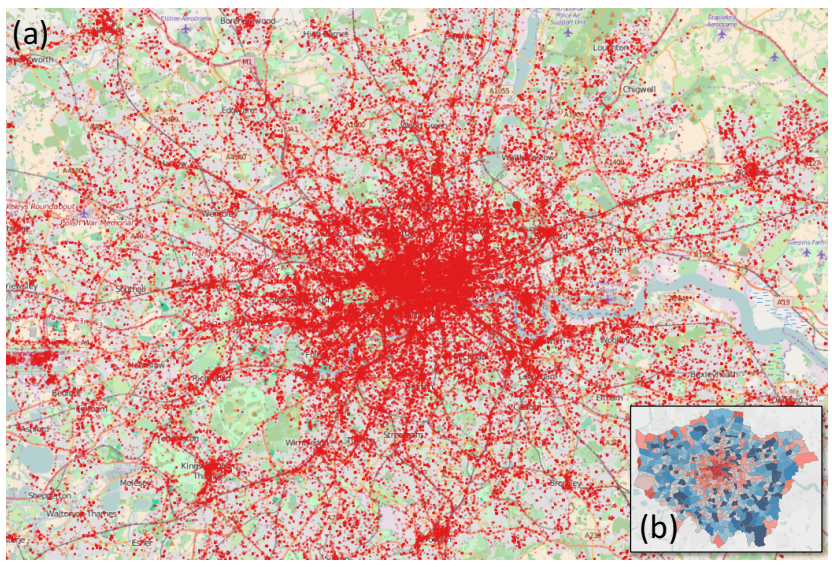

Fig. 1: Twitter data in $5000 \mathrm{~km}^{2}$ area centred on London: (a) 0.4 million geo-tagged data, (b) 0.6 million at ward level.

cell deployment and the BS data is operator-specific. Alternative methods that are operator-neutral have been prototyped in recent years include using mobile apps to mine device data usage [7]. However, both of these methods require largescale participation from smartphone users, which is difficult to achieve without financial incentives.

On the other hand, large volumes of real time online social network (OSN) data can provide network operators with an opportunity to analyze and combine into existing cell planning and operational practices. The advantage of using social media data over operators own data are as follows: 1) it can discover the overall traffic demand across all wireless networks (RATs) and operators (i.e., the whole market, as opposed to its own), 2) it can uncover textual data about how people feel about various aspects of service (not analysed in this paper), and 3) it provides updated data demand: current base stations were deployed by the operator many years ago, and new data trends will have emerged due to changes in the city.

To the best of our knowledge, mapping traffic patterns using OSN data has not been studied in academia (see industrial progress [8]) and the closest example is using OSN data to infer channel occupancy in cognitive radio [9] and customer complaints [10], [11]. In this paper, the authors demonstrate that structured OSN data can act as a superior predictor for long-term and short-term data demand. The paper is organised as follows. In Section III, the authors briefly explain the data sets and analyze the correlation between them. In Section III. the authors demonstrate the ability for Twitter data to predict long-term traffic data and also forward predict shortterm traffic demand. Furthermore, the authors show that the operator- and technology-neutral short-term peak demand can be accurately predicted using long-term statistical parameters. 


\section{Data Sets and Basic Correlations}

\section{A. Data Sets and Metrics}

In this particular paper, the authors utilize four data sets:

1) Structured Social Media Data: 0.6 million geo-tagged Tweets purchased from Twitter over a period of 2 weeks (time resolution in seconds) for the Greater London and surrounding suburbs area $(40 \mathrm{~km}$ radius from centre of London). An example of the Twitter data is mapped in Fig. 17 with ward level aggregate in Fig. 1p.

2) 3G Mobile Traffic Data: 1.2 million mobile phone usage records - Uplink (UL) and downlink (DL) usage, data is aggregated to the ward level. The 3G packetswitched data traffic load (demand) is obtained from the core network over a period of 2 weeks, the time resolution of the data is on a minute basis.

3) 3G Base Station Data: BS location data for an operator.

4) Registered Population Data: ward population and geographical data from the 2011 UK census [12]. The data includes both residential (number of people per household) and business workforce data (number of employees per company).

In order to compare between data sets, the coefficient of determination $R^{2}$ is used. It is a number that indicates how well the statistical regression model fits the data, or in other words: the percentage of variance explained by the model. For a data vector $y=\left[y_{1}, y_{2}, \ldots y_{K}\right]$ (with mean $\bar{y}$ ) and a predicted data vector using the regression model $\hat{y}$, the residue vector is defined as $e=y-\hat{y}$. The coefficient of determination $R^{2}$ is defined as $R^{2} \equiv 1-\frac{\sum_{k} e_{k}^{2}}{\sum_{k}\left(y_{k}-\bar{y}\right)^{2}}$. Adjusted $R^{2}$ is used in this paper to take into account of additional fit parameters $P$, where adjusted $R^{2}=1-\left(1-R^{2}\right) \frac{K-1}{K-P-1}$.

\section{B. Correlations for Existing Data}

The population data of residents and businesses (see Fig. 2a) is widely used in traditional cell planning and spectrum purchases to both gauge the number of potential subscribers and estimate the long-term traffic demand. The data is an important input to deciding where BSs are deployed (see Fig. 2p). The paper first examines the correlation between macro BSs with existing registered population data from the UK census in order to establish a baseline. Fig. 22 shows a scatter plot of the spatial correlation of population density versus BS density in all the London wards. The regression result shows that there is a weak spatial correlation (adjusted coefficient of determination, $R^{2}=0.24$ ), indicating that other factors such as the number of commuters/tourists, historical mobile usage levels, and the local signal propagation account for the remaining $76 \%$ of the variance in BS deployment densities. Using the $3 \mathrm{G}$ traffic data, Fig. $2 \mathrm{~d}$ shows a scatter plot of the spatial correlation between $3 \mathrm{G}$ traffic load (demand) versus both population and BS density in London wards. The results show that there is weak spatial correlation (adjusted $R^{2}=0.29$ ) between the density of BSs and the $3 \mathrm{G}$ traffic demand, and an adequate spatial correlation (adjusted $R^{2}=0.57$ ) between the census population data and the $3 \mathrm{G}$ traffic demand. We compare outlier wards to gain better understanding on why the traffic data

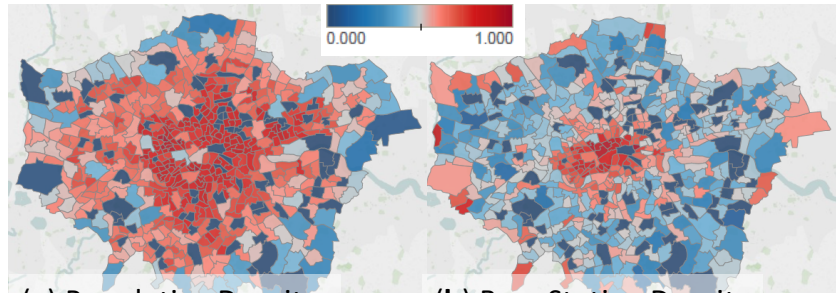

(a) Population Density

(b) Base Station Density

(c)

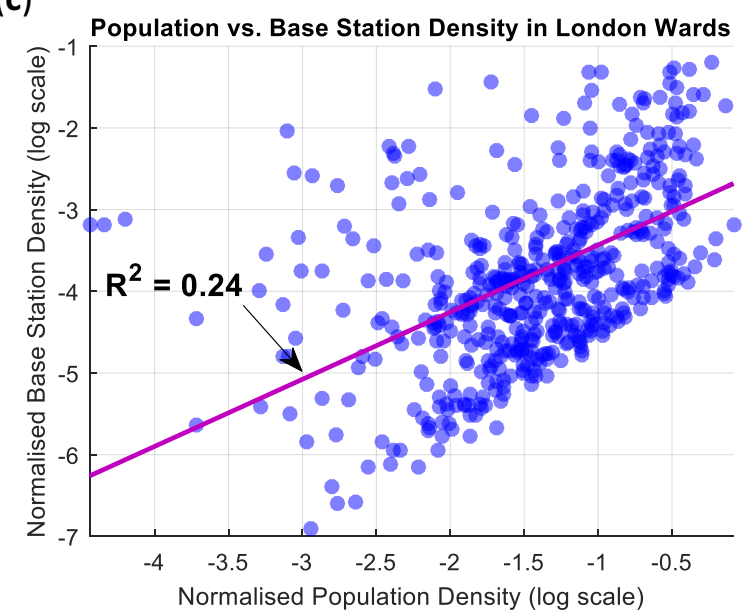

(d)

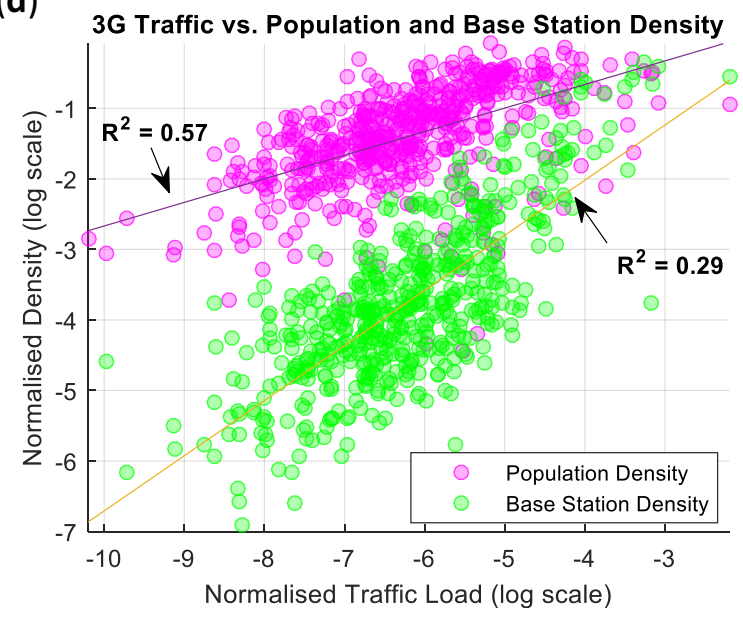

Fig. 2: Normalised density in London Wards of (a) population density, (b) Base Station (BS) density, and scatter plots of: (c) population and BS density, and (d) 3G traffic load (Demand) vs. population and BS density.

doesn't correlate well with the BS density (c) and population density (d). We construct a rank of the densities and compare the rank difference. We discover one particular ward (outlier wards) that has the largest rank difference between traffic and both BS density and population density: Marylebone High Street. The area is host to a number of major commercial streets (bounded by Oxford Street in the south) and tourist attractions (Sherlock Holmes and Madame Tussauds to the north). The traffic demand is ranked one of the highest, and yet both the working and residential population (5th lowest) and BS density are of the lowest. This indicates that existing macro BSs are not well deployed to meet the current traffic 


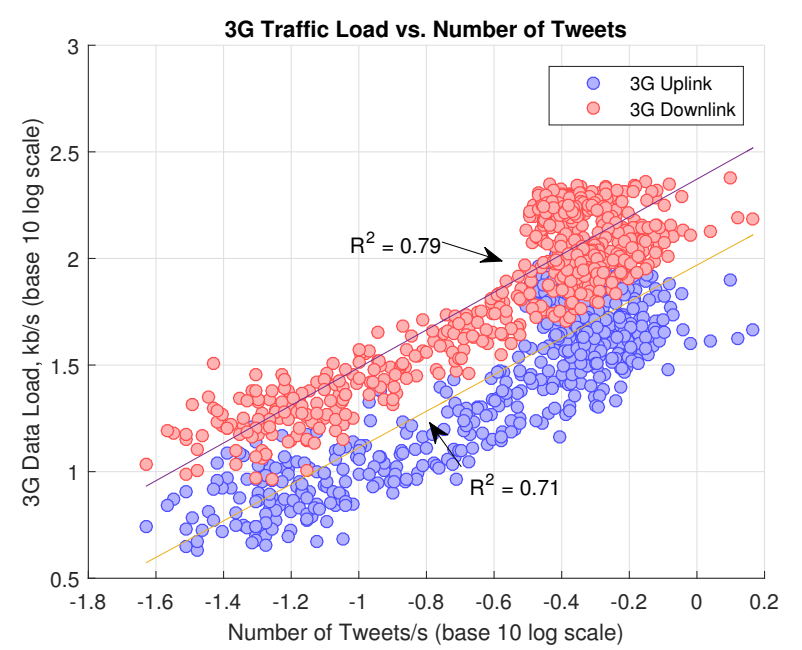

Fig. 3: Spatial Correlation of average 3G Traffic Load (Demand) vs. Number of Tweets.

patterns, and static population census data alone cannot act as an accurate predictor for both the long-term traffic demand or the short-term traffic temporal patterns. In the following sections, the authors will examine how Twitter data can be used to provide a better estimate for long- and short-term traffic demand.

\section{Traffic Demand Estimation Using Twitter}

\section{A. Long-Term Spatial Traffic Demand Estimation}

One of the key metrics that drive small cell deployment is the expected traffic load (demand) in its small coverage area. For deployment of small cells, the short-term temporal variation of the traffic demand is of less interest than the long-term spatial variation. In order to obtain high spatial resolution data, the authors plot the spatial traffic demand pattern against the Twitter activity intensity over the Greater London area. The hypothesis is that Twitter activity level can be used as a proxy for estimating the real wireless traffic demand in both the UL and DL channels. There has already been studies which show that the number of Tweets is highly correlated with the number of people in confined spaces (i.e., a stadium or an airport) [13]. Therefore, the authors in this paper go a step further and infer the traffic demand directly. The data available for analysis from Twitter records only UL Tweets, which consumes negligible bandwidth. Therefore, it is not immediately obvious on why small volumes of UL data should be representative of overall UL and DL data demand, especially given the variety of multimedia and social media applications. Yet, the authors hypothesize that Twitter activity is closely related to other multimedia activities, simply because average human behaviour associates Twitter uplink with all other mobile activities. In fact, in this section the authors show that Twitter activity level is highly correlated with both the UL and DL traffic demand.

In Fig. 3, the ward average 3G data load (UL and DL) is plotted against the number of Tweets per second. Each data point represents a ward, which approximately equals the

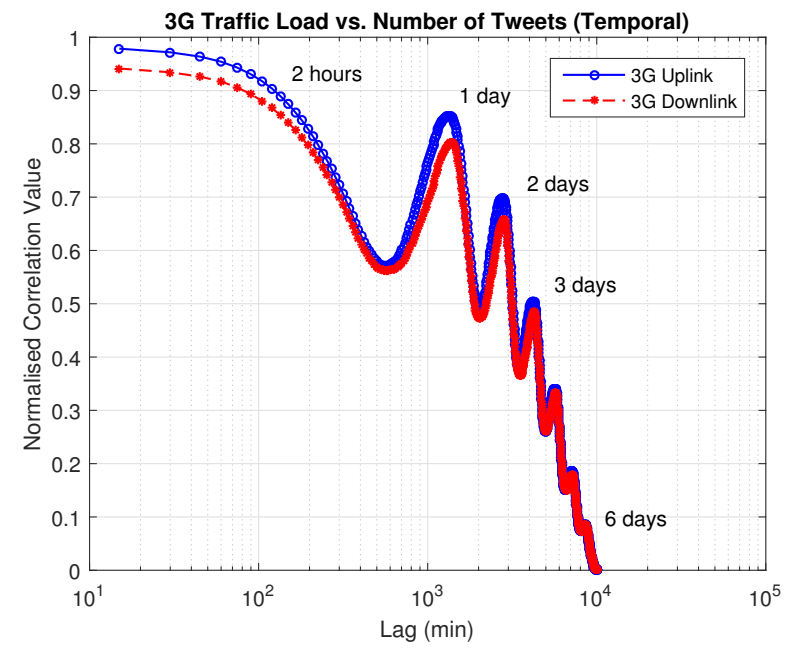

Fig. 4: Temporal Correlation of average 3G Traffic Load (Demand) vs. Number of Tweets over 7 Days.

coverage area of a BS. The results show that a log-linear relationship exists between estimated traffic load $\hat{r}$ (kbps) and the Twitter activity level $n$ (Tweets/s):

$$
\log _{10}\left(\hat{r}_{i}\right)=a_{i} \log _{10}(n)+b_{i}
$$

where $\left[a_{\mathrm{UL}}=0.86 \mathrm{~kb} /\right.$ Tweet $\left.b_{\mathrm{UL}}=1.97 \mathrm{kbps}\right]$, and $\left[a_{\mathrm{DL}}=\right.$ $0.88 \mathrm{~kb} /$ Tweet, $\left.b_{\mathrm{DL}}=2.37 \mathrm{kbps}\right]$. Alternatively, this can be expressed as a power law: $\hat{r}_{i}=10^{b_{i}}(n)^{a_{i}}$. A polynomial least-squares regression is used with the minimum number of parameters $P$ that maximizes the adjusted $R^{2}$ value, as increasing the parameters $P$ will trade-off improved accuracy vs. a decreased adjusted $R^{2}$ value. The correlation achieved is high: $R^{2}=0.71$ for UL and $R^{2}=0.79$ for DL. In other words, the regression model on Twitter data explains $71-79 \%$ of the variations in the $3 \mathrm{G}$ traffic data. The supports our initial hypothesis that OSN data can be used as a more reliable predictor for traffic demand than the census data. Furthermore, OSN data is operator neutral and radio-accesstechnology (RAT) neutral, potentially giving insight on all mobile customers. In general, it is also worth noting that the data rate in UL and DL is fairly low, showing that whilst the aggregate demand is increasing rapidly, the average demand per second remains below the capacity of current BSs. The outlier wards are ones with high average traffic loads and correspond to tourism hotspots in the wards situated in the Westminster and City of London boroughs.

In terms of caveats, the analysis in the paper has only utilized on geo-tagged Tweets. That is to say, we do not understand how the total number of Tweets correlate with the traffic demand, since most Tweets do not have an accurate location (approximately 1\% of Tweets are geo-tagged in the London area). Whilst an increasing number of Tweets and other OSN data are becoming geo-tagged, most data can not be used to identify the traffic pattern. For long-term traffic prediction, historical Twitter and OSN data is more than sufficient. Over several weeks and a large area, this paper was able to show with 0.6 million Tweets that a power 


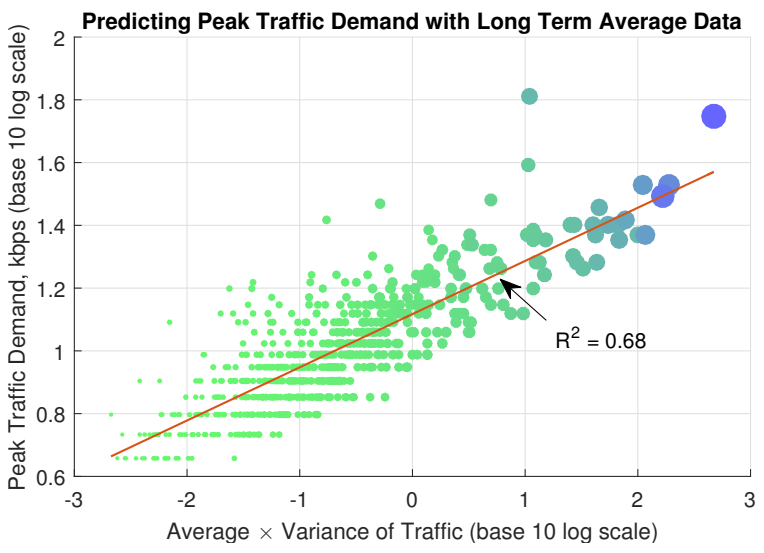

Fig. 5: Predicting Short Term Peak Traffic Demand with LongTerm Statistics.

law relationship between existing geo-tagged Tweets and data demand. However, how accurately can this data be used to predict short-term traffic demand is less clear.

\section{B. Short-Term Temporal Traffic Demand Estimation}

As mentioned previously, another challenge is whether the current Twitter data can be used to accurately predict upcoming short-term traffic demand (i.e., either in a few hours time or the same time a few days later). This has strong applications in self-organising-network (SON) operations [14], such as load balancing. In all of these cases, the upcoming short-term temporal patterns in the traffic demand are of interest. By using cross-correlation between Twitter intensity $n[k]$ (complex conjugate $n *[k]$ ) and the $3 \mathrm{G}$ traffic load $r[k]$ defined as $\sum_{m=-N}^{+N} n *[m] r[m+k]$ with lag $m$ (minutes), the authors examined the normalised correlation value. Fig. 4 shows the normalised cross-correlation value against the lag value. The results show that for a lag of approximately 120 minutes, the correlation is strong $(>0.9)$, meaning that the traffic can be accurately predicted for the next 2 hours using current Twitter activity level. At the same time the next day, the correlation remains strong $(>0.85)$, but this value falls on the third day to 0.7 and on the fourth day to 0.5 . The correlation trend is very similar for UL and DL traffic. That is to say, current Twitter data can be used to predict the UL and DL traffic for the next 2 hours and for the same time on the next day. However, future traffic prediction will need continuous monitoring of geo-tagged Twitter data, which can be expensive to obtain.

\section{Predicting Short-Term Peak Demand}

Previously, the authors have shown that a constant stream of OSN data is needed to obtain up to date high temporal resolution traffic statistics. Nonetheless, there are short-term traffic attributes (i.e., peak demand) that are of interest and can be predicted by using long-term statistics (i.e., the mean and variance). The authors are motivated to use OSN Twitter data to estimate peak demand in order to be operator- and RAT-neutral. In Fig. 5, the authors attempt to predict the peak
Twitter activity level for a particular time period $t\left(n_{\text {peak }, t}\right)$, by showing that the peak demand is log-linear related to the product of the mean $\bar{n}$ and variance $\sigma_{n}^{2}$ of the OSN activity level, such that a least-squares linear regression would yield:

$$
\log _{10}\left(n_{\text {peak }, t}\right)=c \log 10\left(\bar{n} \times \sigma_{n}^{2}\right)+d,
$$

where $c=0.17$ and $d=1.11$ with a strong correlation (adjusted $R^{2}=0.68$ ). Using the relationship in Eq. (1) developed between social media activity level $n$ and the real traffic demand $r$, one can predict the peak traffic demand: $r_{\text {peak }, t}=10^{b_{i}}\left[10^{d}\left(\bar{n} \sigma_{n}^{2}\right)^{c}\right]^{a_{i}}$. The estimated peak traffic demand can be used to avoid service outages by offloading users ahead of time to small cells and neighbouring macro BSs.

\section{CONClusions}

Social media data presents service providers with an unprecedented opportunity to better understand end user consumption patterns and design better systems. In this paper, the authors have shown that the large volumes of real time Twitter data allows for a scalable way to create accurate maps of mobile data demand. In particular, an empirical relationship between the number of Tweets and the data demand is found for uplink and downlink channels, which enables accurate forward traffic prediction up to 2 hours on the same day and for the same time period over the following 1-2 days.

\section{REFERENCES}

[1] X. Chu, D. Lopez-Perez, Y. Yang, and F. Gunnarsson, Heterogeneous Cellular Networks. Cambridge, UK: Cambridge University Press, 2013.

[2] D. Lee, S. Zhou, X. Zhong, Z. Niu, X. Zhou, and H. Zhang, "Spatial modeling of the traffic density in cellular networks," IEEE Wireless Communications, vol. 21, no. 1, pp. 80-88, Mar. 2014.

[3] S. Zhou, D. Lee, B. Leung, X. Zhong, H. Zhang, and Z. Niu, "On the spatial distribution of base stations and its relation to the traffic density in cellular networks," IEEE Access, vol. 3, pp. 998-1010, Mar. 2015.

[4] M. Laner, P. Svoboda, S. Schwarz, and M. Rupp, "Users in Cells: a Data Traffic Analysis," in IEEE Wireless Communications and Networking Conference (WCNC), 2012.

[5] W. Guo and S. Wang, "Interference-aware self-deploying femto-cell," IEEE Wireless Communications Letters, vol. 1, pp. 609-612, Nov. 2012.

[6] W. Guo, S. Wang, X. Chu, Y. Chen, H. Song, and J. Zhang, "Automated Small-Cell Deployment for Heterogeneous Cellular Networks," IEEE Communications Magazine, vol. 51, pp. 46-53, may 2013.

[7] Q. Yu, J. Erman, A. Gerber, Z. Mao, J. Pang, and S. Venkataraman, "Identifying diverse usage behaviors of smartphone Apps," in ACM SIGCOMM, 2011.

[8] I. Barcia, "Radio Challenges and Opportunities for Large Scale Small Cell Deployments," KEIMA, Technical Presentation, 2012.

[9] K. Kotobi, P. Mainwaring, C. Tucker, and S. Bilen, "Data-Throughput Enhancement Using Data Mining-Informed Cognitive Radio," Electronics, vol. 4, pp. 221-238, 2015.

[10] T. Qiu, J. Feng, Z. Ge, J. Wang, J. Xu, and J. Yates, "Listen to Me if You can: Tracking User Experience of Mobile Network on Social Media," in ACM Internet Measurement Conference (IMC), 2010.

[11] K. Takeshita, M. Yokota, and K. Nishimatsu, "Early network failure detection system by analyzing Twitter data," in IEEE International Symposium on Integrated Network Management (IM), 2015.

[12] ONS. (2011) Uk census data 2011. [Online]. Available: http://www. ons.gov.uk/ons/guide-method/census/2011/census-data/index.html

[13] F. Botta, H. S. Moat, and T. Preis, "Quantifying crowd size with mobile phone and Twitter data," Royal Society Open Science, May 2015.

[14] A. Sathiaseelan, M. S. Seddiki, S. Stoyanov, and D. Trossen, "Social SDN: Online Social Networks Integration in Wireless Network Provisioning," in ACM Proceedings of SIGCOMM, 2014. 